\title{
Driver behaviour in unexpected critical events and in repeated exposures - a comparison
}

\author{
Ola Benderius • Gustav Markkula • Krister Wolff • \\ Mattias Wahde
}

Received: 21 May 2012 / Accepted: 4 June 2013 / Published online: 3 July 2013

(C) The Author(s) 2013. This article is published with open access at SpringerLink.com

\begin{abstract}
Purpose This paper aims to determine how truck driver steering behaviour seen in repeated exposures to a critical event correlates to the behaviour resulting from an unexpected exposure to the same event.

Methods Test subjects were exposed to an unexpected critical event in a high-fidelity driving simulator. Next, a slightly modified version of the scenario was repeated several times for each subject. The driver behaviour was then analysed using standard statistical tests.

Results It was found that, in general, drivers keep most of their steering behaviour characteristics between test settings (unexpected and repeated). This is particularly interesting since a similar kind of behaviour preservation is generally not found in the case of braking behaviour. In fact, only one significant difference was found between the two test settings, namely regarding time-to-collision at steering initiation.

Conclusions In experiments involving both an unexpected event and several repeated events one can, at least in some cases, design the repeated event such that behavioural data collected from that setting can be used along with data from the unexpected setting. Using this procedure, one
\end{abstract}

O. Benderius $(\varangle) \cdot K$. Wolff $\cdot$ M. Wahde

Department of Applied Mechanics,

Chalmers University of Technology, Göteborg, Sweden

e-mail: ola.benderius@chalmers.se

K. Wolff

e-mail: krister.wolff@chalmers.se

M. Wahde

e-mail: mattias.wahde@chalmers.se

G. Markkula

Volvo Technology Corporation, Göteborg, Sweden

e-mail: gustav.markkula@volvo.com can significantly increase the amount of collected data, something that can strongly benefit, for example, driver modelling.

Keywords Driver behaviour - Repeated exposures . Evasive steering $\cdot$ Driving simulator $\cdot$ Driver modelling

\section{Introduction}

Road traffic accidents constitute a large problem on a global scale. Apart from great economical and social costs, the accidents also cause a significant number of injuries and deaths. The number of worldwide fatalities have recently been estimated at over one million per year [15], of which $8 \%$ occur in Europe. Driver behaviour is widely considered as a contributing factor in many road traffic accidents. Therefore, research efforts regarding the safety aspects of such behaviour have been intensified during recent years [9].

As a way to understand and further study the impacts of driver behaviour, models that capture aspects of driver behaviour are being developed (for a recent review, see [12]). An important goal of driver behaviour research is to find simple phenomenological relations that explain driver behaviour in certain situations. Examples of such behaviour could, for instance, be braking behaviour as a function of headway to an obstacle [8], or steering behaviour as a function of perceptual inputs [14].

Data used in the development of a driver model are typically collected from real-world driving experiments or driving simulator studies. In most experimental arrangements, the intention is to mimic realistic scenarios in order to measure realistic behavioural responses in drivers. However, fully realistic scenarios cannot easily be accomplished 
in a non-naturalistic driving experiment. In particular, in order to obtain sufficient amounts of data for statistical analysis and development of driver models, it might be necessary to expose each driver to the studied scenario multiple times. This is so since, for economical and other reasons, one often cannot involve a very large number of drivers in an experiment. Furthermore, for driver model development, in view of the individual differences between drivers, one generally needs quite many data points from each driver in order for meaningful model development to be possible. Before making use of the results obtained from such experiments one must therefore first understand the effects (if any) of repeated exposures to the scenario under study.

Previously, several studies have considered driver reaction times in braking scenarios [5]. It has been shown that, when reacting to expected (e.g. repeated) events, drivers' brake reaction times are reduced significantly $[2,10]$. Furthermore, it has been concluded that test subjects brake earlier and more strongly in repeated events [10]. However, it has also been shown that some behavioural aspects might be preserved in repeated exposures [2]. In this paper, the effects on driver steering behaviour caused by repetition of a critical event are studied.

The data used for the analysis presented in this paper were collected in a truck simulator study regarding driver behaviour in connection with an electronic stability control (ESC) system. In the experiment, the truck drivers were asked to drive on a road in a winter environment, where they were exposed to an unexpected critical lead vehicle braking scenario, inducing a rapid steering manoeuvre. In a brief pre-study, the unexpected scenario was not found to be reliable in inducing sufficient ESC-relevant (loss of control) data, and the scenario was therefore repeated several times for each driver.

The analysis presented here will be centred on the effects of steering behaviour caused by the repeated exposures to the scenario rather than the effects on driver behaviour caused by the ESC system. Furthermore, only events in which the driver successfully evaded a collision with the lead vehicle will be considered. Events involving a collision should be studied separately since the typical driver behaviour appears to be fundamentally different, in particular for the unexpected case.

In order to ascertain the validity of steering data collected from repeated exposures, a statistical comparison between aspects of the unexpected and the repeated data has been carried out. Four different criteria (C1-C4) for measuring the validity of repeated exposure data have been defined as:

C1 Scenario tuning: Can the repeated scenario be tuned so that the steering manoeuvre is initiated under similar conditions in both test settings (unexpected and repeated)? If so, when the truck driver initiates the evasive steering, there are no crucial differences in road position, speed, or headway to the lead vehicle, regardless of the test setting (unexpected or repeated).

C2 Manoeuvre similarity: Is the steering manoeuvre carried out in a similar fashion in both settings, that is without crucial differences in maximum steering wheel angles, steering wheel rates, or steering wheel reversal rates? ${ }^{1}$

C3 Preservation of individual behaviour: Do the test subjects keep their individual steering behaviour characteristics between the two test settings?

C4 Effects of learning: Are there no crucial effects of learning on how steering avoidance is carried out, meaning that test subjects do not change their steering performance over repetitions?

\section{Method}

Data were collected in a high-fidelity moving-base truck simulator at the Swedish National Road and Transport Research Institute (VTI). The simulator uses a moving base platform providing lateral movement as well as roll and pitch rotations. A visual system provides a $105^{\circ}$ field of view using forward-facing projectors, and emulated rear mirrors using monitor screens. For the study considered in this paper, a six-wheeled rigid truck with a wheel base of $6.2 \mathrm{~m}$ (from first to last axle) was simulated.

\subsection{Simulator experiment}

\subsubsection{Scenario}

The main scenario used in the experiment was originally proposed in [2] and involves a critical lead vehicle braking scenario, as illustrated in Fig. 1. The scenario took place on a divided four-lane motorway with two lanes in each direction. The road speed limit for trucks was $80 \mathrm{~km} / \mathrm{h}$. While the test subject was driving at speed $v_{1}$ in the right lane, a passenger car, referred to as the principal other vehicle (POV), overtook the truck at speed $v_{2}=R_{x} v_{1}$ using the left lane, where $R_{x}>1$ is a constant. At a time headway $T_{\text {cut }}$ with respect to the truck, the POV (at lateral speed $v_{\text {cut }}$ ) changed from the left to the right lane. Once in the right lane, the POV continued forward, still at speed $v_{2}$. Then, without any apparent reason, at time headway $T_{b}$, the POV braked strongly with the constant deceleration $d_{b}$. The deceleration continued until the POV stopped completely (with one exception, described in the next paragraph). Right before the deceleration, the speed was instantaneously set to $v_{1}$ in

\footnotetext{
${ }^{1}$ Steering wheel reversal rates are explained in Section 2.2.2.
} 
order to ensure that the time headway was always less than, or equal to, $T_{b}$. When the critical scenario was initiated, the road friction was lowered from $\mu_{1}$ to $\mu_{2}$ in order to emulate a slippery road surface.

As seen in Table 1, the scenario was parametrized for three different versions: (i) unexpected avoidance (UA), (ii) repeated avoidance (RA), and (iii) catch trial (CT), i.e. a version in which no steering avoidance was needed. In the $\mathrm{CT}$, the POV deceleration ended at speed $v_{3}$ and was then followed by an acceleration $a_{\text {acc. }}$. The CT was included so that the drivers would not be certain regarding the nature of a repeated event, i.e whether it was an RA scenario or a CT scenario.

The parameters for the RA scenario were chosen so that the test subjects would initiate their steering approximately at the same time in the repeated events as they did in the unexpected scenario. In order to meet this requirement, a larger deceleration $d_{b}$ was used in the repeated events and in the catch trials so as to compensate for reduced reaction times [5], and the speed $v_{3}$ was used so as to control the time at which the test subjects initiated evasive steering. Appropriate parameter values were chosen based on results from

\section{a}

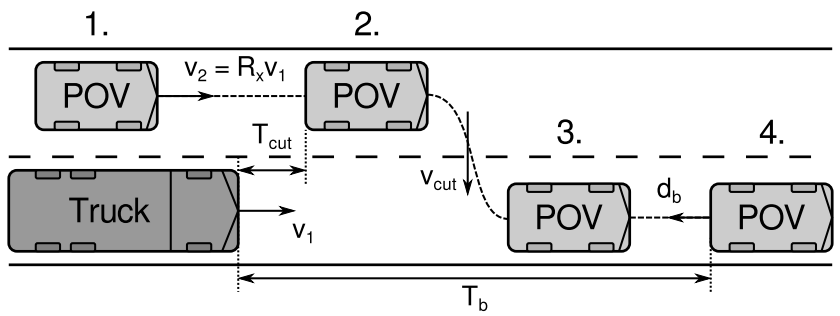

b

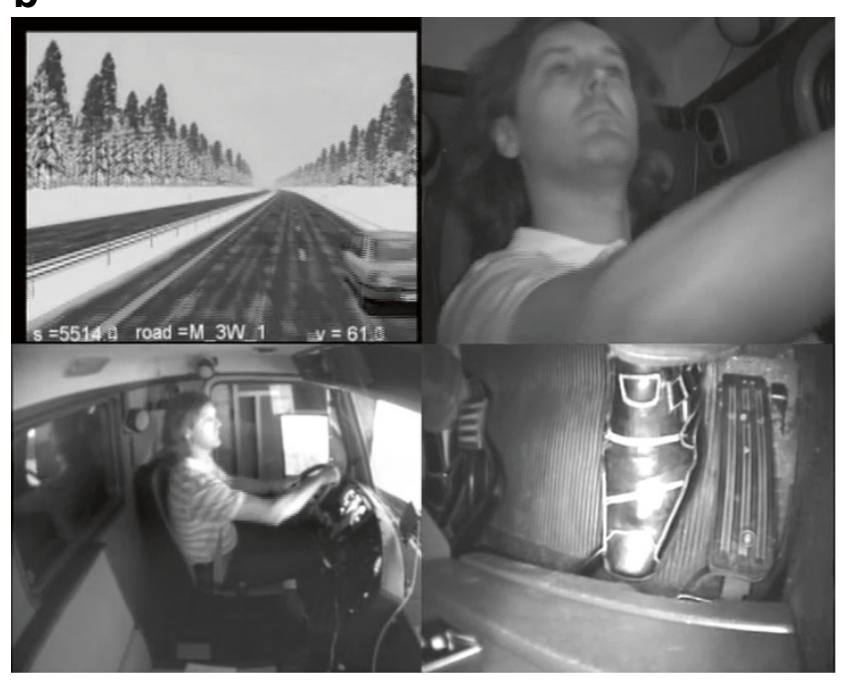

Fig. 1 Top panel: The critical lead vehicle braking scenario where a passenger car overtakes the test subject's truck. The numbers 1-4 denote different points in time. Bottom panel: A snapshot from the experiment. Here, one of the test subjects is in the process of avoiding the POV in the unexpected avoidance scenario
Table 1 Parameters for the critical lead vehicle braking scenario

\begin{tabular}{llll}
\hline Parameter & UA & RA & CT \\
\hline$R_{x}$ & 1.15 & 1.15 & 1.15 \\
$T_{\text {cut }}$ & $0.9 \mathrm{~s}$ & $0.9 \mathrm{~s}$ & $0.9 \mathrm{~s}$ \\
$v_{\text {cut }}$ & $5.4 \mathrm{~km} / \mathrm{h}$ & $5.4 \mathrm{~km} / \mathrm{h}$ & $5.4 \mathrm{~km} / \mathrm{h}$ \\
$T_{b}$ & $1.5 \mathrm{~s}$ & $1.5 \mathrm{~s}$ & $1.5 \mathrm{~s}$ \\
$d_{b}$ & $0.35 \mathrm{~g}$ & $0.45 \mathrm{~g}$ & $0.45 \mathrm{~g}$ \\
$\mu_{1}$ & 0.7 & 0.7 & 0.7 \\
$\mu_{2}$ & 0.25 & 0.25 & 0.25 \\
$v_{3}$ & - & - & $45 \mathrm{~km} / \mathrm{h}$ \\
$a_{\text {acc }}$ & - & - & $0.3 \mathrm{~g}$ \\
\hline
\end{tabular}

$\mathrm{UA}, \mathrm{RA}$, and CT refer to unexpected avoidance, repeated avoidance, and catch trial versions of the scenario, respectively. See the main text for the parameter definitions

(i) a brief pre-study in a fixed base simulator, and (ii) computer simulations using a simple driver model. Both the UA and RA events were tuned such that evasive braking alone was insufficient to avoid a collision.

\subsubsection{Experimental procedure}

The experiment consisted of three parts with a preceding training session. The last part, involving a double lane change on a cone track, will not be discussed in this paper. The training session, inspired by [7] and [13], included driving in steady-state traffic as well as a few repetitions of non-critical braking and steering exercises. The total length of the training session was about ten minutes and it was carried out on the same simulated road as the critical scenario (described above).

In the first part of the experiment, the test subjects were instructed to drive normally at $80 \mathrm{~km} / \mathrm{h}$ on the motorway described above. After approximately four minutes of driving, including four overtaking vehicles and a non-critical double lane change induced by a road construction site, the UA scenario occurred. Unknown to the test subjects, half of them had the ESC system present in their trucks, and half did not.

In the second part of the experiment, the subjects were informed about the ESC system and whether or not it was present in their vehicle (explained as an "anti-skid system"). They were also instructed about the RA and CT scenarios (described above), and that both scenarios would be repeated several times in a random order. The drivers were then asked to drive at $80 \mathrm{~km} / \mathrm{h}$ on the same motorway as in the previous part, and to apply evasive steering only when they considered it required in order to avoid a collision (i.e. only in the RA event).

During the second part, each subject experienced, in random order, four overtaking vehicles, six occurrences of the 
RA scenario, and eight occurrences of the CT scenario. Note that, in no case did the CT scenario evoke a critical steering avoidance behaviour, and the analysis below thus concerns only the RA scenario in relation to the UA scenario.

\subsubsection{Test subjects}

In total, 24 test subjects participated in the experiment. The drivers were divided into two equally large groups based on driving experience (low or high). The drivers belonging to the low-experience group were mainly recruited from a local driving school, where they soon would get their licence for truck-trailer combinations. However, all lowexperience drivers already had a driver's licence for the rigid truck simulated in the experiment. The drivers belonging to the high-experience group were mainly recruited from local hauler companies.

\subsection{Experimental design}

The steering manoeuvre was divided into four time intervals, as illustrated in Fig. 2. The first interval, $I_{1}$, starts at time $t_{0}$ when the overtaking car initiates braking, and ends at time $t_{1}$ when the truck driver initiates evasive braking. The second interval, $I_{2}$, starts at time $t_{1}$, and ends at time $t_{2}$ when the truck driver initiates evasive steering. The interval $I_{3}$ starts at time $t_{2}$, and ends at time $t_{3}$ when the truck driver has reached the maximum steering wheel angle to the left. Finally, the interval $I_{4}$ starts at time $t_{3}$, and ends at time $t_{4}$ when the truck driver reaches the largest steering wheel angle to the right. Time $t_{4}$ is given as the first (in time) local minimum at which the steering wheel angle signal reached a value less than $-20^{\circ}$, time $t_{3}$ is given as the time of the largest steering wheel angle between $t_{0}$ and $t_{4}$, and time $t_{2}$ is given as the time of the first steering wheel angle value below $8^{\circ}$ when tracking the signal backwards from $t_{3}$. Note that, for the schematic illustration in Fig. $2, t_{4}$ could have

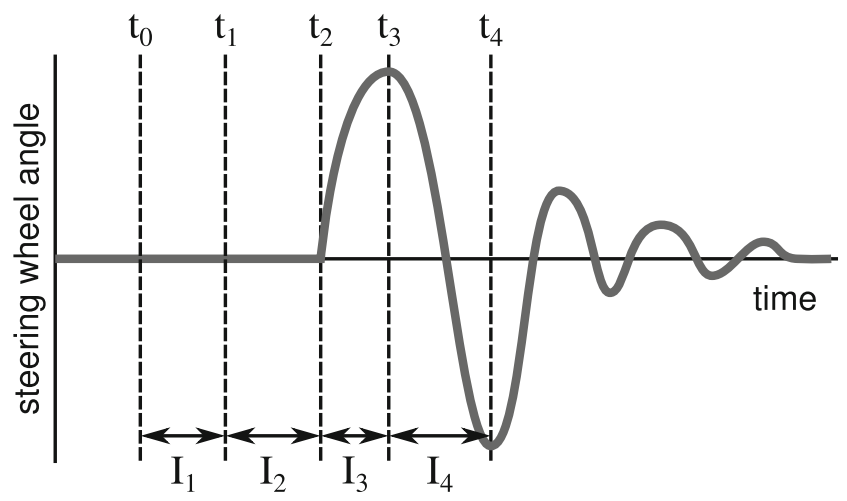

Fig. 2 A schematic illustration of a steering manoeuvre required to avoid a collision in the lead vehicle braking scenario. The overtaking vehicle starts to brake at time $t_{0}$, and the truck driver initiates braking at time $t_{1}$

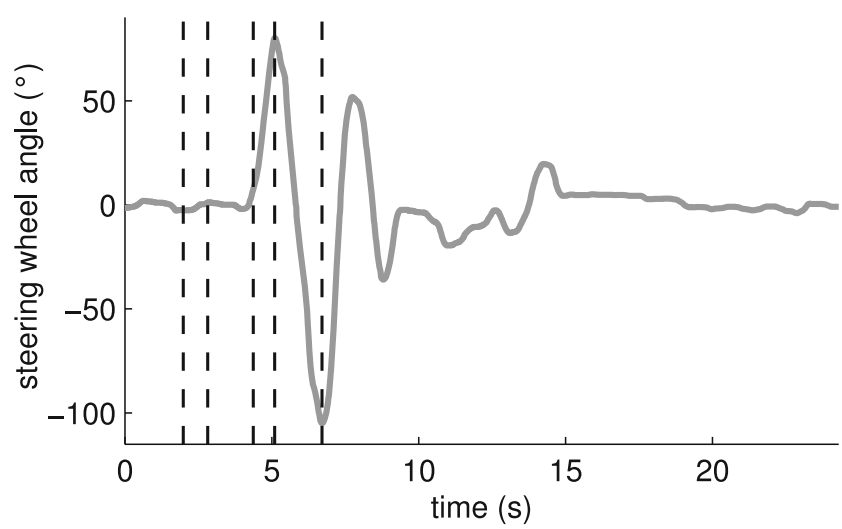

Fig. 3 A typical steering signal extracted from actual driving data. The vertical lines denote the time intervals described in Fig. 2

be defined as the global minimum of the steering wheel angle. However, some steering manoeuvres involved a control loss, in which case subsequent minima of the steering wheel angle could be deeper than the first minimum, hence the definition of $t_{4}$ given above. An actual steering response is presented in Fig. 3.

\subsubsection{Independent variables}

The independent variables considered in this paper are: test setting (UA or RA), and repetition (one through six).

\subsubsection{Dependent variables}

A number of dependent variables, presented in Table 2, were extracted from each event in the driving data. Time to collision (TTC) is measured as the time it would take for the truck to cover the distance between the front of the truck and the rear of the POV (assuming current POV and truck speeds, i.e. disregarding any accelerations). The steering wheel reversal rate (SWRR), was defined as the number of steering wheel reversals per minute, larger than a certain minimum angular value [11].

Table 2 The different measures analysed

\begin{tabular}{llll}
\hline Measure & Source & Criteria & Normal \\
\hline TTC & $t_{2}$ & $\mathrm{C} 1, \mathrm{C} 3, \mathrm{C} 4$ & Yes \\
lateral position & $t_{2}$ & $\mathrm{C} 1, \mathrm{C} 3, \mathrm{C} 4$ & No \\
longitudinal speed & $t_{2}$ & $\mathrm{C} 1, \mathrm{C} 3, \mathrm{C} 4$ & Yes \\
steering wheel angle & $t_{3}, t_{4}$ & $\mathrm{C} 2, \mathrm{C} 3, \mathrm{C} 4$ & No, No \\
steering wheel rate & $I_{3}, I_{4}$ & $\mathrm{C} 2, \mathrm{C} 3, \mathrm{C} 4$ & No, No \\
SWRR $\left(5^{\circ}\right)$ & $I_{3}, I_{4}$ & $\mathrm{C} 2, \mathrm{C} 3, \mathrm{C} 4$ & No, Yes \\
\hline
\end{tabular}

The data sources are presented in Fig. 2. The list of criteria is presented at the end of Section 1. Normality was tested for each variable using the Shapiro-Wilk test 


\subsubsection{Statistical tests}

Two types of tests were used when testing for significant differences in dependent variables between test settings (i.e. criteria $\mathrm{C} 1$ and $\mathrm{C} 2$ ). If the samples of a dependent variable were found to be normally distributed a dependent t-test for paired samples was used, otherwise a Wilcoxon signed-rank test was used. Normality was tested using the Shapiro-Wilk test, as reported in Table 2. For each test subject and dependent variable, the unexpected event as well as the mean of all repeated events are used as one pair of values. Furthermore, for each significance test, the Pearson's correlation coefficient $r$ was calculated as a measure of effect size. The use of the paired samples t-test or the Wilcoxon test implies that no assumption regarding the homogeneity of variances needs to be made [4].

Pearson's correlation coefficient is also used for two other purposes, first to determine the paired sample correlation of a dependent variable between test settings, in order to see whether driver behaviour is transferred between settings (C3) and second to determine the correlation of a dependent variable between repetitions, in order to study learning effects (C4).

Three of the four criteria involve an absence of a crucial difference in behaviour. Here, a crucial difference is defined as a medium effect size $(|r|>0.3)$ [1].

\section{Results}

According to the experimental set-up, the maximum amount of data was 24 repetitions of the unexpected event, and 144 repetitions of the repeated event. Data from one of the repeated events were lost due to experimental problems. In eight unexpected and two repeated events, the test subjects

Table 3 The top row shows the theoretical upper limit on the amount of data, based on the experimental design, whereas the second row shows the actual amount of data collected

\begin{tabular}{lccc}
\hline & Unexpected & Repeated A & Repeated B \\
\hline Design & 24 & 144 & 144 \\
Acquired & 24 & 143 & 143 \\
Steering & 16 & 141 & 94 \\
No collision & 9 & 88 & 36 \\
Filtered & 8 & 84 & 33 \\
\hline
\end{tabular}

The third row shows the number of events in which the driver initiated steering (i.e. applied a steering wheel angle above $15^{\circ}$ ), the fourth row shows the number of events where the driver successfully avoided a collision, and the bottom row shows the amount of data after filtering (see the main text for a description). The middle column includes repetitions from all drivers whereas the right column only includes repetitions collected from drivers represented in the left column
Table 4 The number of repetitions used for each of the test subjects presented in the last row of the last column of Table 3

\begin{tabular}{llllllllr}
\hline Test subject & 1 & 2 & 3 & 4 & 5 & 6 & 7 & 8 \\
\hline Repetitions & 3 & 5 & 3 & 5 & 6 & 5 & 3 & 3 \\
\hline
\end{tabular}

For each test subject, the means of the variables extracted from corresponding repetitions are used in the t-tests

did not attempt any evasive steering at all. A successful evasive manoeuvre (i.e. without collision) occurred in 9 of the unexpected events and 88 of the repeated events. As stated in the end of Section 1, all events where a collision occurred were discarded. Four other repeated events were discarded since the test subject violated the instructions by initiating steering directly when the POV began braking. Finally, data from one unexpected event were discarded since the driver steered much earlier compared to the other test subjects (with a TTC of 7.9 s). In Table 3, Repeated $A$ refers to all repetitions matching the corresponding row criterion, while Repeated $B$ only includes matching repetitions that also share a test subject represented in the left column. In Table 4, the 33 filtered data points in Repeated $B$ are matched to their corresponding test subject. Figure 4 summarizes the TTC at steering initiation for the retained data points after filtering.

In the rest of this section, the steering manoeuvre will be analysed by comparing the dependent variable pairs extracted from the unexpected events with the variables from the repeated events. In order to make it possible to use a paired sample t-test, all repeated events from the same test subjects were merged by calculating the mean of each dependent variable. Therefore, $2 \times 8$ values will be compared in each t-test.

In Figs. 5, 6, 7, 8 and 9, the left panel compares the unexpected and averaged repeated scenarios, whereas the right panel compares the repetitions over repetition number. The left panel includes the eight sample pairs used in the t-tests,

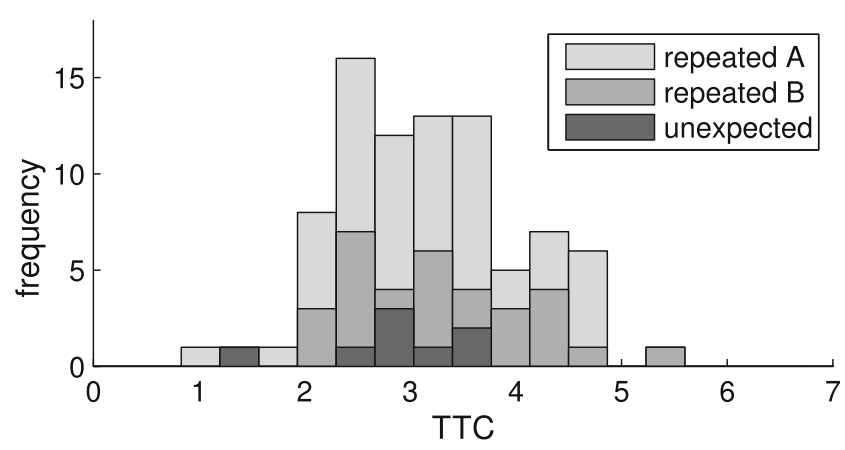

Fig. 4 The TTC at steering initiation of all unexpected and repeated events remaining after filtering (see Table 3) 

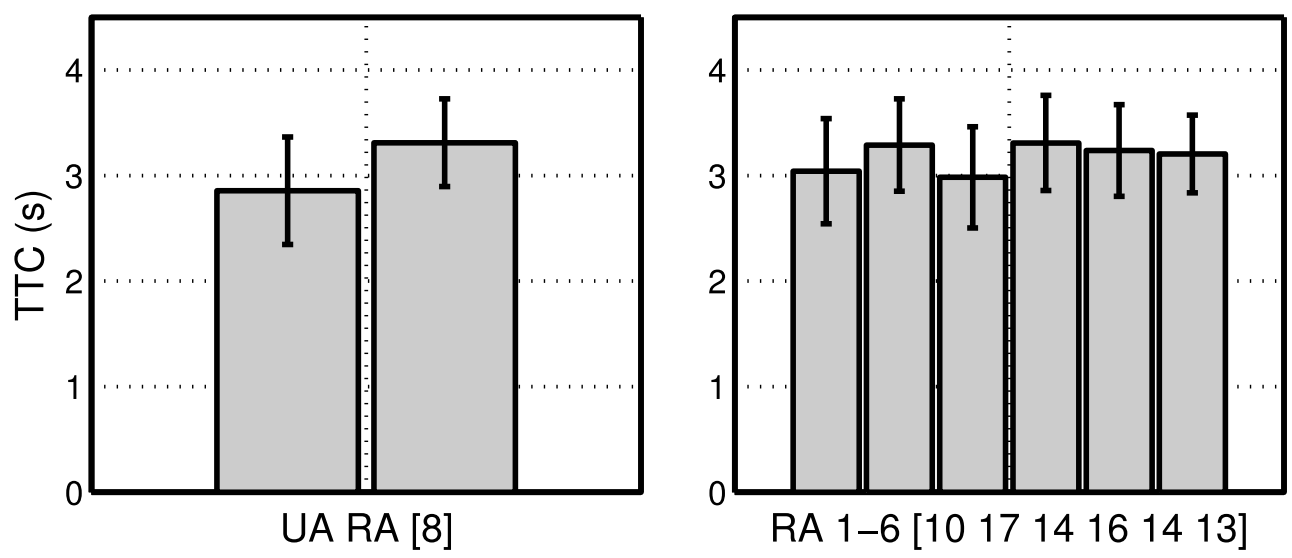

Fig. 5 The TTC at time $t_{2}$ (steering initiation)

and the right panel includes the 84 repetitions presented in the middle column of Table 3. Error bars show $95 \%$ confidence intervals, calculated under the assumption of a normal sampling distribution.

\subsection{Situation at steering initiation}

When analysing the situation at steering initiation $\left(t_{2}\right)$ between test settings, a significant difference was found in one out of three dependent variables: By using the t-test, the significant difference was found in TTC (see Fig. 5) between UA $(M=2.86, S D=0.74)$ and RA $(M=3.31$, $S D=0.60) ; t(7)=-2.4, p<0.05, r=0.67$. No significant difference was found in lateral position (see Fig. 6) or longitudinal speed.

\subsection{Steering manoeuvre}

The steering manoeuvre was analysed by comparing six of the presented (see Table 2) dependent variables between the UA and the RA. The six variables include: (i) maximum

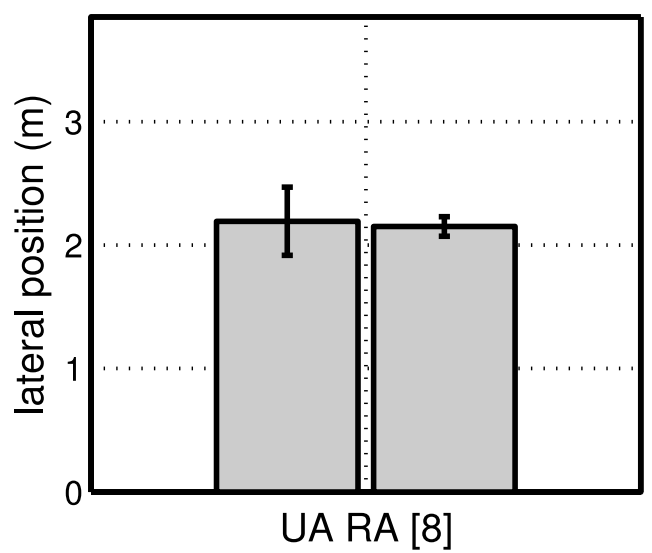

steering wheel angle to the left ( $\left.t_{3}\right)$, (ii) maximum steering wheel angle to the right $\left(t_{4}\right)$, (iii) maximum steering wheel rate while turning left $\left(I_{3}\right)$, (iv) maximum steering wheel rate while turning right $\left(I_{4}\right)$, (v) SWRR $\left(5^{\circ}\right)$ while turning left $\left(I_{3}\right)$, and (vi) SWRR $\left(5^{\circ}\right)$ while turning right $\left(I_{4}\right)$. No significant differences were found between the two test settings (UA and RA) for any of the variables. However, one of the variables, namely steering wheel rate in the interval $I_{4}$ (see Fig. 8) gave a considerably larger effect size ( $p>0.05$, $r=-0.59)$ compared to the other five variables $(p>0.05$, $|r| \leq 0.30)$. Two of the other variables, steering wheel angle at time $t_{4}(p>0.05, r=-0.25)$ and SWRR $\left(5^{\circ}\right)$ in the interval $I_{3}(p>0.05, r=-0.30)$, are exemplified in Figs. 7 and 9 respectively.

\subsection{Preservation of steering behaviour}

By calculating a paired sample correlation, using the same $2 \times 8$ values as above, one can study the preservation of steering behaviour between both test settings. When testing all the nine dependent variables listed in Table 2, it was

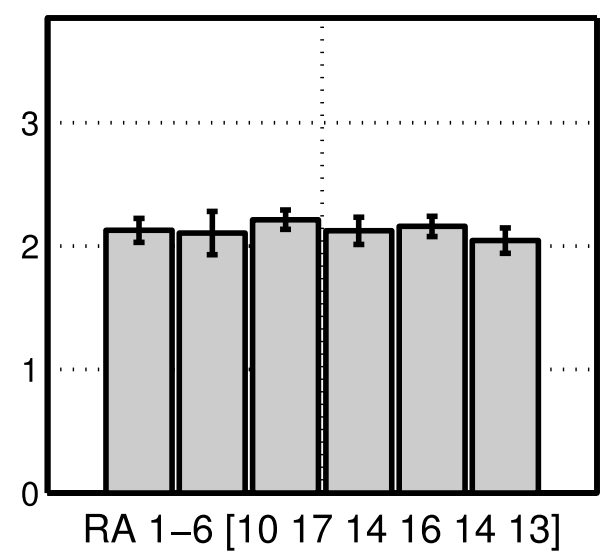

Fig. 6 The lateral position at time $t_{2}$ (steering initiation) 

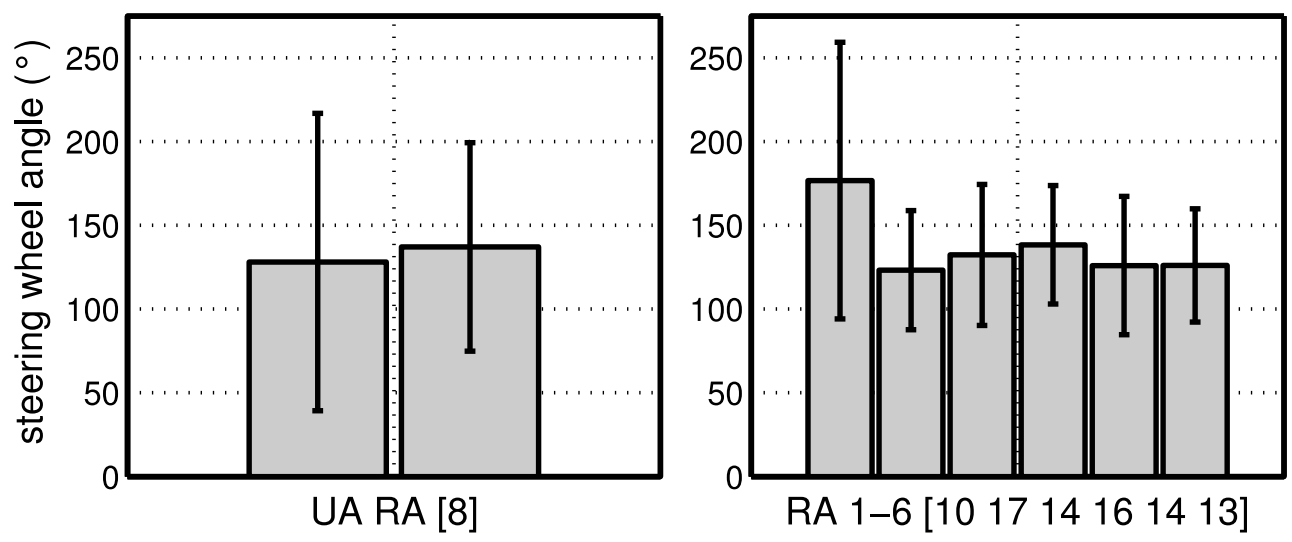

Fig. 7 The steering wheel angle at time $t_{4}$ (maximum steering wheel angle to the right)

found that four of them had a significant correlation, namely steering wheel angle at times $t_{3}(p<0.05, r=0.94)$ and $t_{4}(p<0.05, r=0.95)$, and steering wheel rate in the intervals $I_{3}(p<0.05, r=0.93)$ and $I_{4}(p<0.05, r=0.97)$. No other variable correlation showed significance, nor any high values of the Pearson's correlation coefficient $r$. In Fig. 10, two examples of paired sample correlation are presented, one with a large $r$ value, and one with a small $r$ value.

\subsection{Learning effects in steering behaviour}

For the analysis of potential learning effects, the data collected in the unexpected scenario were not used. Therefore, since the use of paired data was not required, the 84 data points presented in the middle column of Table 3 could be used. In this case, a strict test of significance would be complicated (but not impossible) since each driver is only represented in a subset of the repetitions, with different subsets for different drivers. Therefore, only the Pearson's correlation coefficient was determined for each dependent variable, and for which a large (absolute) $r$ value would indicate a learning effect.

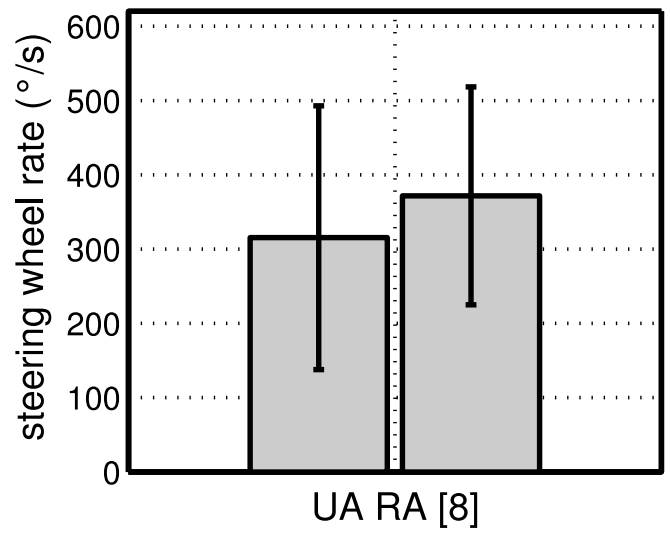

By calculating Pearson's correlation coefficient for each dependent variable, it was found that no large absolute $r$ values existed. For three of the values, the modulus exceeded 0.1 , namely SWRRs in the intervals $I_{3}(r=-0.12$; see Fig. 9) and $I_{4}(r=0.19)$, and steering wheel angle at time $t_{4}(r=0.11$; see Fig. 7$)$.

\section{Discussion}

The discussion is structured as follows: First the findings in Section 3 will be related to the criteria listed at the end of Section 1. Then, aspects of the experimental setup will be discussed, focusing on repeated critical events for collecting steering behaviour data. Finally, the general applicability of such data will be discussed in the context of driver modelling.

\subsection{Criteria}

The validity of the first criterion $(\mathrm{C} 1)$ regarding scenario tuning, cannot be entirely confirmed: When drivers initiated evasive steering, (at time $t_{2}$ ), there was a significant

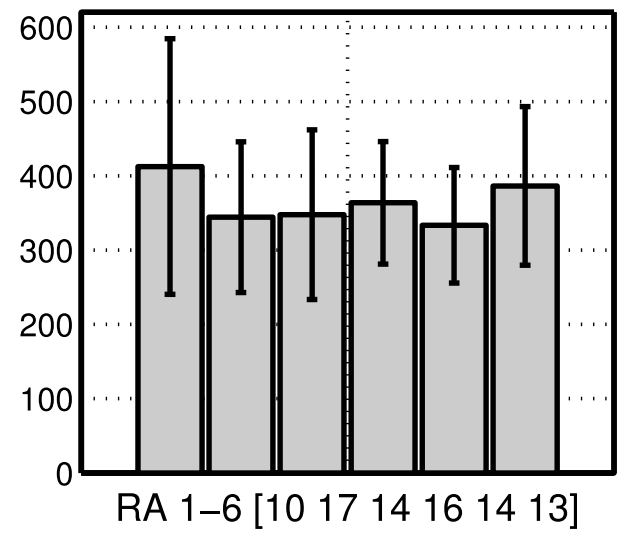

Fig. 8 The maximum steering wheel rate in interval $I_{4}$ (between maximum steering wheel angle to the left, and maximum steering wheel angle to the right) 


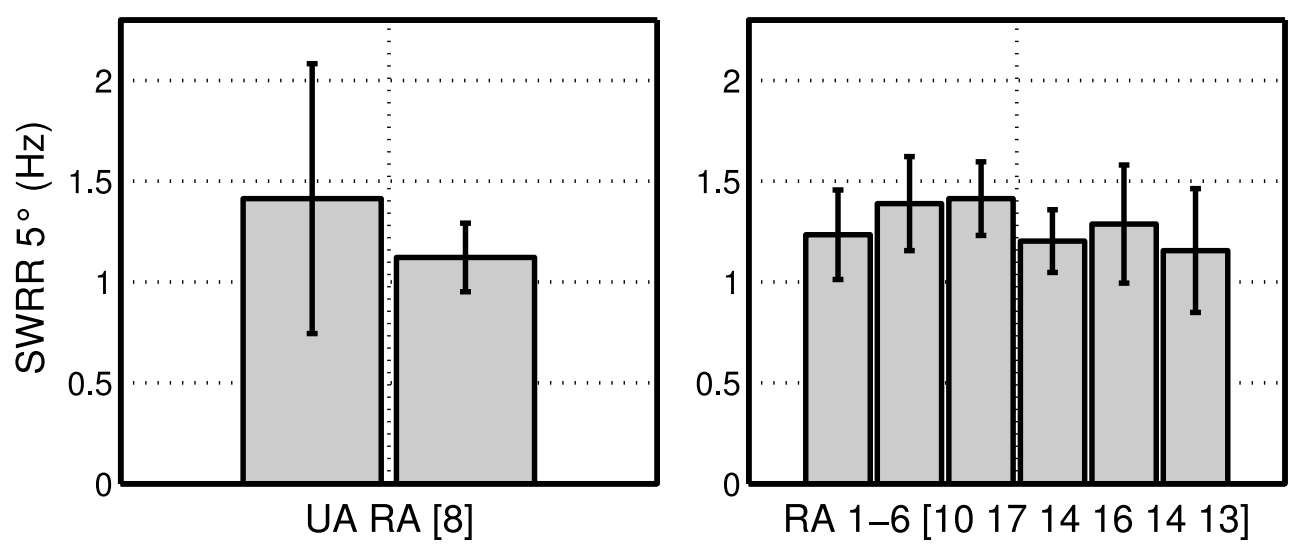

Fig. 9 The $5^{\circ}$ SWRR in interval $I_{3}$ (between steering initiation, and maximum steering wheel angle to the left)

difference in TTC $(p<0.05, r=0.67)$, such that initiation occurred, on average, $0.5 \mathrm{~s}$ earlier in the repeated events, resulting in a slightly less critical situation. However, even though the difference is significant and with a large effect size, it might be argued that the difference is sufficiently low for $\mathrm{C} 1$ to be fulfilled, since drivers appear to behave similarly during the remainder of the UA and RA scenarios (as discussed below).

At the time of steering initiation, there was no significant difference in lateral position or speed between test settings. For lateral position, one could intuitively assume that test subjects would position their trucks further to the left in a repeated event. No such tendencies were seen, however.

The validity of criterion $\mathrm{C} 2$, regarding manoeuvre similarity, can be confirmed, as there were no significant differences between any analysed steering wheel angles, steering wheel rates, or SWRRs. There is, however, an apparent difference (not significant) regarding steering wheel angle at time $t_{4}$ (see Fig. 7) in the very first repetition. The same phenomenon can be seen in the steering wheel rate during the interval $I_{4}$ (see Fig. 8). A possible explanation could be that test subjects overestimate the severity of the situation in the first repetition, and then regain a behaviour similar to the one seen in the unexpected event.

Also criterion $\mathrm{C} 3$, regarding preservation of individual behaviour appears to be valid. Using paired sample correlation, it was shown that test subjects keep their steering characteristics very well ( $r$ values close to 1$)$ regarding maximum steering wheel angles and steering wheel rates. They do not, however, preserve their characteristics regarding SWRRs. Arguably, the criterion can still be considered to be valid, since SWRRs are less important compared to maximum steering wheel angles and steering wheel rates when considering the outcome of the steering behaviour, i.e. avoiding the collision. It should also be pointed out that there were, as stated for the previous criterion, no significant differences in SWRRs between test settings on the population level.

Interestingly, from Fig. 10 one can see that drivers tend to have a lower SWRR value $(1 \mathrm{~Hz})$ in the repeated events compared to the unexpected events. A possible explanation, if one considers experience to be scenario-specific, could be related to the fact that inexperienced drivers (in this case meaning drivers that have not yet been exposed to the critical event) in general have a larger SWRR compared to
Fig. 10 The correlation of two different dependent variables, namely steering wheel rate in the interval $I_{4}$, and SWRR $\left(5^{\circ}\right)$ in the interval $I_{3}$. The first had the largest Pearson's correlation coefficient $(r=0.97)$ of all dependent variables, and the second had the smallest coefficient $(r=0.03)$ a

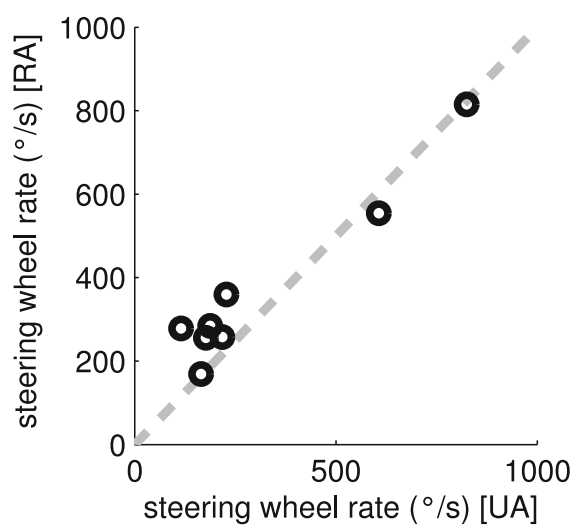

b

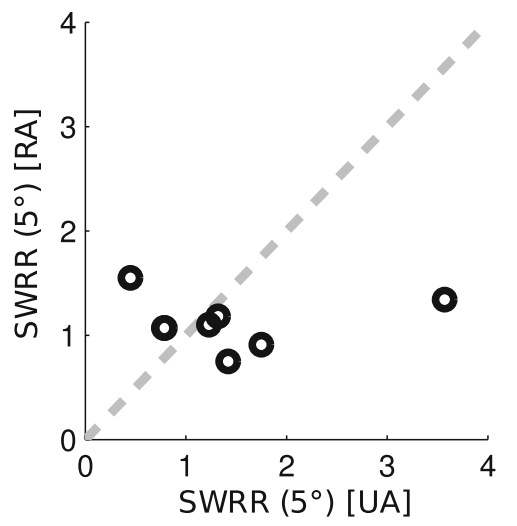


those drivers who have experienced the critical event [3]. In order to explain the observed phenomenon in terms of control, one can hypothesize that an experienced (in the scenario) driver carries out the steering manoeuvre in an open-loop manner, rather than in a closed-loop manner [6]. However, as mentioned above, even though a difference in SWRR between test settings seems probable, no statistically significant difference was found.

Finally, the validity of criterion $\mathrm{C} 4$, regarding (the absence of) learning effects can be confirmed: There were no crucial $(r>0.3)$ effects of learning for any of the dependent variables. There were some small effects of learning for the SWRRs ( $r=-0.12$, see Fig. 9, and $r=0.19)$, and the maximum steering wheel angle to the right $(r=0.11)$. However, such small effects would hardly have any bearing here, considering the small number of repetitions.

It should also be pointed out that there were no large effects of learning on lateral position $(r=-0.07)$. Again, one would intuitively assume that drivers should tend to gradually keep further to the left over repetitions. However, no such behaviour was seen.

\subsection{Experimental set-up}

In general, and especially when considering critical situations, the amount of available data is limited. Furthermore, when collecting data from critical situations, there are problems involving safety, cost, and test subject expectancy.

By using multiple repetitions of a critical event, the number of acquired data points can be strongly increased. Furthermore, by using repeated events, one can reduce costs by decreasing the total number of test subjects as well as increasing the efficiency (event rate) during the experiment. Safety problems can also be addressed by using repetitions since it allows for test subjects to be well instructed during the experiment.

However, repetitions typically require some additional work. First, the repeated scenario must be designed to compensate for known effects on driver behaviour. For instance, it was concluded in [10] that driver braking behaviour is largely affected by repetitions, in particular by decreased reaction times and stronger braking. In the case considered here, a significant difference between test settings was found in the TTC at steering initiation. In retrospect, this difference could perhaps have been eliminated by further tuning the parameters for the RA scenario. Secondly, the data collected from repeated scenarios must be validated for the application at hand. For this purpose, it is highly recommended first to collect data from an unexpected version of the scenario under study, as was done in the experiment considered here, and then to make a comparative analysis between the data collected in the unexpected scenario and the repeated tests. Another reason for including unexpected events is that the behaviour observed in repeated events is probably only a subset of all possible types of behaviour for the given scenario.

\subsection{Applicability in driver modelling}

Since all four criteria (C1-C4) were found to be valid, possibly with the partial exception of $\mathrm{C} 1$, it has been shown that the steering behaviour collected in repeated events can be used in driver modelling, at least for the case of successful evasive manoeuvres. As seen in Table 3, only data from 8 out of 24 drivers were used in the comparison. From the remaining 16 drivers: (i) eight did not attempt steering at all, (ii) seven applied steering but still collided with the POV, and (iii) one steered much earlier compared to the other drivers. By using the data analysed in this paper, one can thus only cover a third of the observed behaviour. All four types of behaviour must be covered when generating a general driver model for the critical scenario under study. However, it should be pointed out that it is likely to be much easier to model those drivers that do not apply any evasive steering (i.e. another third of the drivers considered in this experiment).

\section{Conclusion}

In this paper, it has been shown that the steering behaviour observed in repeated critical scenarios to a large extent preserves the characteristics of the steering behaviour found in an unexpected critical scenario, making it possible to use data from repeated scenarios in, for example, driver modelling for evasive steering.

It has been demonstrated that a repeated scenario can be tuned so that evasive steering is initiated under the same conditions as in an unexpected scenario. Furthermore, it was found that there were no significant differences in lateral position and speed between test settings. There was, however, a significant difference in TTC ( $p<0.05, r=0.67)$, but it was argued that the difference did not seem to change the rest of the observed steering behaviour. No significant differences were found in the maximum steering wheel angles or the steering wheel rates during the manoeuvre itself.

It was also found that test subjects keep their steering characteristics between test settings. Very high correlation values ( $r$ close to 1$)$ were obtained from paired sample correlation tests of maximum steering wheel angles and maximum steering wheel rates. Furthermore, no large effects of learning (measured using Pearson's correlation coefficient) were found in the collision avoidance manoeuvre.

Using repeated events in an experiment is recommended when large amounts of data are needed, for example when developing driver models. However, it is also recommended 
to combine the repeated events with an unexpected event in order to make it possible to validate the data collected in the repeated events.

Acknowledgments This work was supported by VINNOVA (the Swedish Governmental Agency for Innovation Systems; grant number 2009-02766) as well as by the competence centre ViP (Virtual Prototyping and Assessment by Simulation, www.vipsimulation.se, co-financed by the VINNOVA grant 2007-03083 and the competence centre partners).

The authors wish to thank Lars Eriksson, Anne Bolling, Jonas Andersson Hultgren, and Anders Andersson at the Swedish National Road and Transport Research Institute, for their invaluable support in the preparation, execution, and analysis phases of this study.

Open Access This article is distributed under the terms of the Creative Commons Attribution License which permits any use, distribution and reproduction in any medium, provided the original author(s) and source are credited.

\section{References}

1. Cohen J (1988) Statistical power analysis for the behavioral sciences. Lawrence Erlbaum

2. Engström J, Aust M, Viström M (2010) Effects of working memory load and repeated scenario exposure on emergency braking performance. Hum Factors: J Hum Factors Ergon Soc 52(5):551559

3. Erséus A (2010) Driver-vehicle interaction: identification, characterization and modelling of path tracking skill. $\mathrm{PhD}$ thesis, KTH

4. Field A (2009) Discovering statistics using SPSS. SAGE publications Ltd
5. Green M (2000) How long does it take to stop? Methodological analysis of driver perception-brake times. Transp Hum Factors 2(3):195-216

6. Hollnagel E, Woods DD (2005) Joint cognitive systems foundations of cognitive systems engineering. CRC Press / Taylor \& Francis

7. Jamson A, Smith P (2003) Are you used to it yet? Braking performance and adaptation in a fixed-base driving simulator. In: Proceedings of the driving simulator conference North America, Dearborn, Detroit, Michigan. 8th-10th Oct 2003

8. Jurecki R, Stańczyk T (2009) Driver model for the analysis of pre-accident situations. Veh Syst Dyn 47(5):589612

9. Lee J (2008) Fifty years of driving safety research. Hum Factors: J Hum Factors Ergon Soc 50(3):521-528

10. Lee J, McGehee D, Brown T, Reyes M (2002) Collision warning timing, driver distraction, and driver response to imminent rearend collisions in a high-fidelity driving simulator. Hum Factors: $\mathbf{J}$ Hum Factors Ergon Soc 44(2):314-334

11. Markkula G (2006) A steering wheel reversal rate metric for assessing effects of visual and cog. secondary task load. In: Proceedings of the ITS world congress, London

12. Markkula G, Benderius O, Wolff K, Wahde M (2012) A review of near-collision driver behavior models. Hum Factors: J Hum Factors Ergon Soc 54(6):1117-1143

13. McGehee D, Lee J, Rizzo M, Dawson J, Bateman K (2004) Quantitative analysis of steering adaptation on a high performance fixed-base driving simulator. Trans Res Part F: Traffic Psychol Behav 7(3):181-196

14. Wann JP, Wilkie RM (2004) How do we control high speed steering? In: Vaina LM, Beardsley SA, Rushton SK (eds) Optic flow and beyond. Kluwer Academic Publishers, Dordrecht, pp 371389

15. World Health Organization (2009) Global status report on road safety: time for action 